\title{
Road condition assessment by OBIA and feature selection techniques using very high- resolution WorldView-2 imagery
}

\begin{abstract}
Accurate information on the conditions of road asphalt is necessary for economic development and transportation management. In this study, object-based image analysis (OBIA) rule-sets are proposed based on feature selection technique to extract road asphalt conditions (good and poor) using WorldView-2 (WV-2) satellite data. Different feature selection techniques, including support vector machine (SVM), random forest (RF) and chisquare (CHI) are evaluated to indicate the most effective algorithm to identify the best set of OBIA attributes (spatial, spectral, textural and colour). The chi-square algorithm outperformed SVM and RF techniques. The classification result based on $\mathrm{CHI}$ algorithm achieved an overall accuracy of $83.19 \%$ for the training image (first site). Furthermore, the proposed model was used to examine its performance in different areas; and it achieved accuracy levels of $83.44,87.80$ and $80.26 \%$ for the different selected areas. Therefore, the selected method can be potentially useful for detecting road conditions based on WV-2 images.
\end{abstract}

Keyword: Object-based image analysis (OBIA); WorldView-2; Feature selection; Chisquare; Road condition 